\title{
Editorial Opinion on the Article: First Record of Ectrodactyly and Polymelia in the Paradox Frog Pseudis Paradoxa (Linnaeus, 1758) From Northern Brazil
}

\author{
Diana $\mathrm{C}^{*}$ \\ University of Oradea, Romania
}

*Corresponding author: Cupsa Diana, University of Oradea, Romania, Tel: 00-40-259-408229;

Email: cupsa2007@yahoo.com

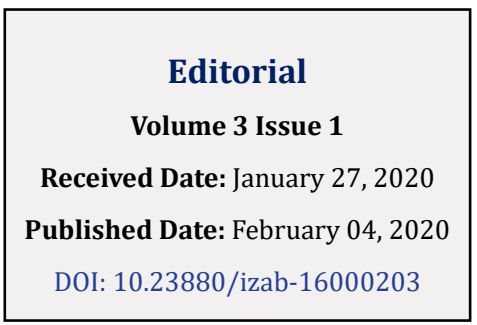

Volume 3 Issue 1

Received Date: January 27, 2020

DOI: $10.23880 /$ izab- 16000203

\section{Editorial}

The article deals with a case of polymelia and ectrodactylia discovered for the first time in paradox frog in the Eastern Amazon. In the actual context of the continuous growth of the human impact on the environment worldwide it raises questions about the causes of these deformities which have to be investigated.

The authors mention that a series of abnormalities which affect the limbs of different anuran species are natural. When the rate of these abnormalities grows above a certain limit, it raises question about the factors involved in their causes.

At the paradox frog these deformities are recorded for the first time and they are of a great interest especially because two different abnormalities occurs at the same specimen. Although only one specimen was found with this type of modification, so it is possible the cause is a mutation with no special external influences.

The authors discuss the possible causes of these abnormalities, which sometimes can be multifactorial. The causes can be internal or external. The internal factors are represented by genetic mutations which in normal condition have a constant rate. The external factors are very diverse they can be physical, chemical or biological.

One of the most important factors which determine the polymelia at different frog species is the parasite trematod worm from genus Ribeiroia. The presence of this parasite at paradox frogs is not known yet. The authors through this record open a new investigation direction to elucidate if this parasite has extended to the paradox frog also and can represent a mutational factor, causing limb morphological abnormalities.

The abnormality determinates a reduced mobility of the specimens and this fact can increase mortality at the affected individuals. Because this fact it is important to elaborate monitoring procedures to be able to determine the number of the specimens form the frog populations affected by this abnormality in order to estimate the effect upon the population effectives.

Another important cause which is known to determine morphological abnormalities of the limbs is represented by the agrochemicals used by the human populations for their crops. In the present days the intensive agriculture practices extend more and more, so the amount of the chemicals which enters the natural environments is growing permanently.

The agricultural caused pollution together with other pollution forms determined by the human activity represent one of the major threatening factors which lead to the decrease of the terrestrial and aquatic environment biodiversity.

The paper is documented with a series of references from scientific papers dealing with the same subject which sustain their findings. They show the limbs modifications represent a widespread problem in different frog species from different regions of the world.

The paper has a practical value in the context of identification of limb malformations in a new region of Brazil in paradox frogs. This finding raises questions on the possible causes of these malformations the region which have to be 
investigated according to the presence or absence of certain chemical and biological mutation factors.

The malformations can be determined by a parasite trematod, in this case we witness the spreading of the parasite to a new host species unknown before. In the case of the confirmation of the new host species it is important to ensure the monitoring of the evolution of the host-parasite complex to be able to evaluate its impact on the parasite frog.
In the case the abnormalities are produced by the chemical pollution this can represent a threat not only for the investigated species but also upon the regional biodiversity. To elucidate this problem, it is necessary to perform future ecotoxicology studies which will determine the amount and the nature of the pollutants. Secondary to the ecotoxicology studies efficient measures can be taken in order to stop and reduce pollution and protect the regional biodiversity. 